\title{
Development and Calibration of a Field-Deployable Microphone Phased Array for Propulsion and Airframe Noise Flyover Measurements
}

\author{
William M. Humphreys, Jr., David P. Lockard†, Mehdi R. Khorrami, \\ William G. Culliton ${ }^{\S}$, Robert G. McSwain \\ NASA Langley Research Center, Hampton, Virginia \\ Patricio A. Ravetta ${ }^{¥}$ \\ AVEC, Inc., Blacksburg, Virginia \\ Zachary Johns ${ }^{\text {r }}$ \\ National Institute of Aerospace, Hampton, Virginia
}

\begin{abstract}
A new aeroacoustic measurement capability has been developed consisting of a large channelcount, field-deployable microphone phased array suitable for airframe noise flyover measurements for a range of aircraft types and scales. The array incorporates up to $\mathbf{1 8 5}$ hardened, weather-resistant sensors suitable for outdoor use. A custom 4-mA current loop receiver circuit with temperature compensation was developed to power the sensors over extended cable lengths with minimal degradation of the signal to noise ratio and frequency response. Extensive laboratory calibrations and environmental testing of the sensors were conducted to verify the design's performance specifications. A compact data system combining sensor power, signal conditioning, and digitization was assembled for use with the array. Complementing the data system is a robust analysis system capable of near real-time presentation of beamformed and deconvolved contour plots and integrated spectra obtained from array data acquired during flyover passes. Additional instrumentation systems needed to process the array data were also assembled. These include a commercial weather station and a video monitoring / recording system. A detailed mock-up of the instrumentation suite (phased array, weather station, and data processor) was performed in the NASA Langley Acoustic Development Laboratory to vet the system performance. The first deployment of the system occurred at Finnegan Airfield at Fort A.P. Hill where the array was utilized to measure the vehicle noise from a number of sUAS (small Unmanned Aerial System) aircraft. A unique in-situ calibration method for the array microphones using a hovering aerial sound source was attempted for the first time during the deployment.
\end{abstract}

\section{Introduction}

$\mathrm{T}$ he recently concluded NASA Environmentally Responsible Aviation (ERA) Project was formulated to explore vehicle concepts and technologies that improve fuel efficiency, reduce noise levels, and decrease harmful emissions of both the current and future fleet of aircraft traversing the national air transportation system. ${ }^{1}$ The ERA project had among its goals the reduction of aircraft noise by 42 cumulative EPNL dB below Stage 4. To meet this goal, a comprehensive portfolio of integrated technology

\footnotetext{
*Aerospace Engineer, Advanced Measurements and Data Systems Branch, Associate Fellow AIAA

${ }^{\dagger}$ Aerospace Engineer, Computational Aerosciences Branch, Senior Member AIAA

¥Aerospace Engineer, Computational Aerosciences Branch, Associate Fellow AIAA

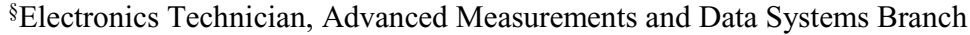

'Aerospace Engineer, Aeronautics Systems Engineering Branch

${ }^{\ddagger}$ Chief Research Engineer, Senior Member AIAA

${ }^{\mathrm{r}}$ Research Test Engineer
} 
demonstrations was developed including wind tunnel and flight testing of noise reduction concepts. ${ }^{2}$ The new NASA Flight Demonstrations and Capabilities (FDC) Project builds upon the success of the ERA Project by promoting focused flight experiments to validate critical technologies, including noise reduction concepts. These flight experiments require the use of measurement diagnostics, both aircraft- and groundbased, in order to quantitatively evaluate the benefit of specific concepts. In the realm of noise reduction characterization, one of the primary tools for such quantitative measurements is the microphone phased array.

NASA Langley Research Center (LaRC) has a long history of successfully utilizing microphone arrays in ground test facilities like the Quiet Flow Facility ${ }^{3}$ as well as in flight test campaigns. In 2006, a 167microphone array was deployed at the NASA Wallops Flight Facility (WFF) to conduct an extensive series of baseline airframe noise measurements on two Gulfstream aircraft. ${ }^{4}$ Figure 1 shows an aerial view of this array deployed on the overrun area of Runway 4 at WFF. The microphones were low-cost, commodity electret units placed on the runway surface in a central mounting plate and on individual ground plates. A highly distributed signal conditioning and data acquisition system was deployed with most of the acquisition hardware housed in ventilated cabinets on the runway near the microphones. While useful measurements were obtained during the 2006 campaign, there were a number of issues with the operational performance of the array that limited its ultimate utility:

- The microphones were not hardened for use in an outdoor environment. As a result of moisture contamination, the sensitivity of the sensors drifted significantly during the test campaign, making accurate quantitative measurements of noise levels difficult. Furthermore, there was no effective method for calibrating the entire array of microphones in-situ.

- The environmental enclosures for the signal conditioning and data acquisition hardware were tall and positioned very close to the perimeter of the array, as can be seen in Fig. 1. This provided multiple acoustic scattering and reflecting surfaces inside the aperture of the array, partially degrading the array performance.

- The environmental enclosures incorporated ventilation and thermoelectric air conditioning. However, these systems generated too much noise near the microphones and were ineffective at sufficiently reducing the temperatures inside the enclosures, causing equipment

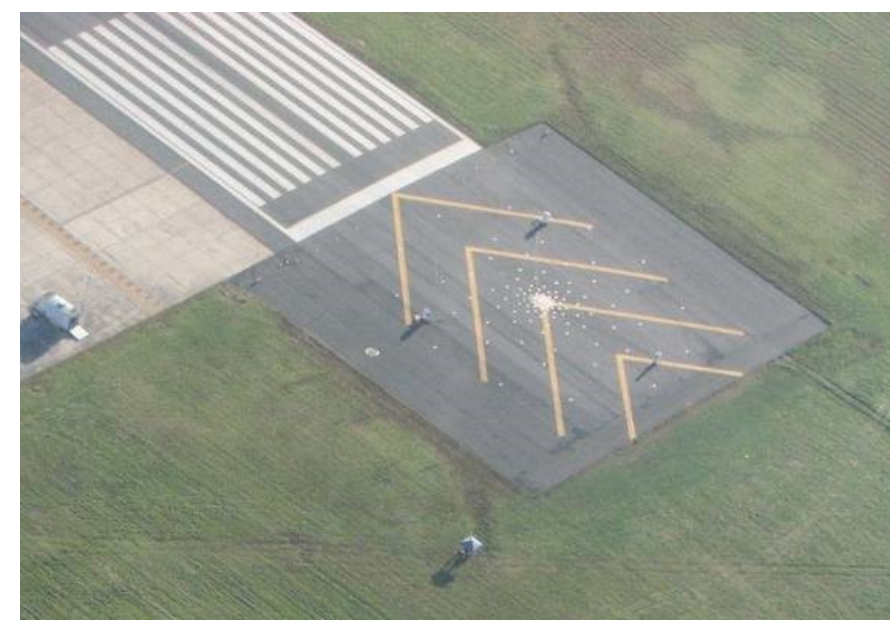

Figure 1. 167-microphone array at NASA WFF in 2006. Dots on overrun area are microphone ground plates. Data acquisition system cabinets are visible around the perimeter of the array. failures during the test campaign.

In order to address the issues listed above, a completely new array design was developed suitable for long-duration outdoor deployment where airframe and propulsive noise measurements are desired for a range of aircraft types and scales. The new array incorporates up to 185 hardened, weather-resistant sensors that use custom 4-mA current loop circuits with temperature compensation to power the sensors over extended cable lengths with minimal degradation of the signal to noise ratio and frequency response. Along with the new sensors, a data system combining sensor power, signal conditioning, and digitization was assembled for use at a central site away from the array to minimize disruptions to the array aperture. This 
paper describes the design, testing, and initial deployment of the new hardware for a sUAS (small Unmanned Aerial System) aircraft test campaign conducted at Fort A.P. Hill in Virginia in the summer of 2015.

\section{Phased Array System}

Microphones: The phased array system utilizes 185 custom hardened and weatherproof microphones designed around a commercially available sensor. An ensemble of strict performance requirements were defined for the sensors based on the needs of the aeroacoustic community when performing measurements of propulsion and airframe noise sources in flight. These requirements include a reasonably flat frequency response over a $20 \mathrm{~Hz}$ to $10 \mathrm{kHz}$ range, a sensitivity of greater than $2 \mathrm{mV} / \mathrm{Pa}$ (i.e., greater than $-54 \mathrm{~dB}$ with respect to $1 \mathrm{~V} / \mathrm{Pa}$ ), a noise floor less than $40 \mathrm{dBA}$, and sensors able to survive submersion in 1 inch of water or more (due to the need for extended outdoor placement of the units). After evaluating several commercially available sensors, the Knowles WP-23849 microphone capsule was chosen as the sensing element for the array ${ }^{*}$. This microphone exhibits a flat frequency response to approximately $8 \mathrm{kHz}$ and is waterproof to 9.8 feet of submersion.

The Knowles microphone is intrinsically a voltage-driven device. However, due to the need to use 400-foot cables to transmit the microphone signals from the array to the central data acquisition system, a custom two-wire, low-noise, 4-mA constant current excitation system was developed to power the sensors. The microphone receiver circuit used to perform this function is shown in Figure 2. The circuit includes temperature compensation via a simple voltage regulator (an LM340) and op amp (an LM6132). Figures 3 and 4 show respectively the front and back sides of the microphones, constructed in-house at LaRC on 3by 3 -inch printed circuit boards (PCB's) and using a LEMO connector to provide power and signal access to the board. A total of 230 microphones of the type shown in Figs. 3 and 4 were fabricated (185 for the array plus spares). In order to improve the hardening of the microphones, the populated PCB's were conformal coated with an acrylic sealant to render the circuitry on the front and back of the boards waterproof.

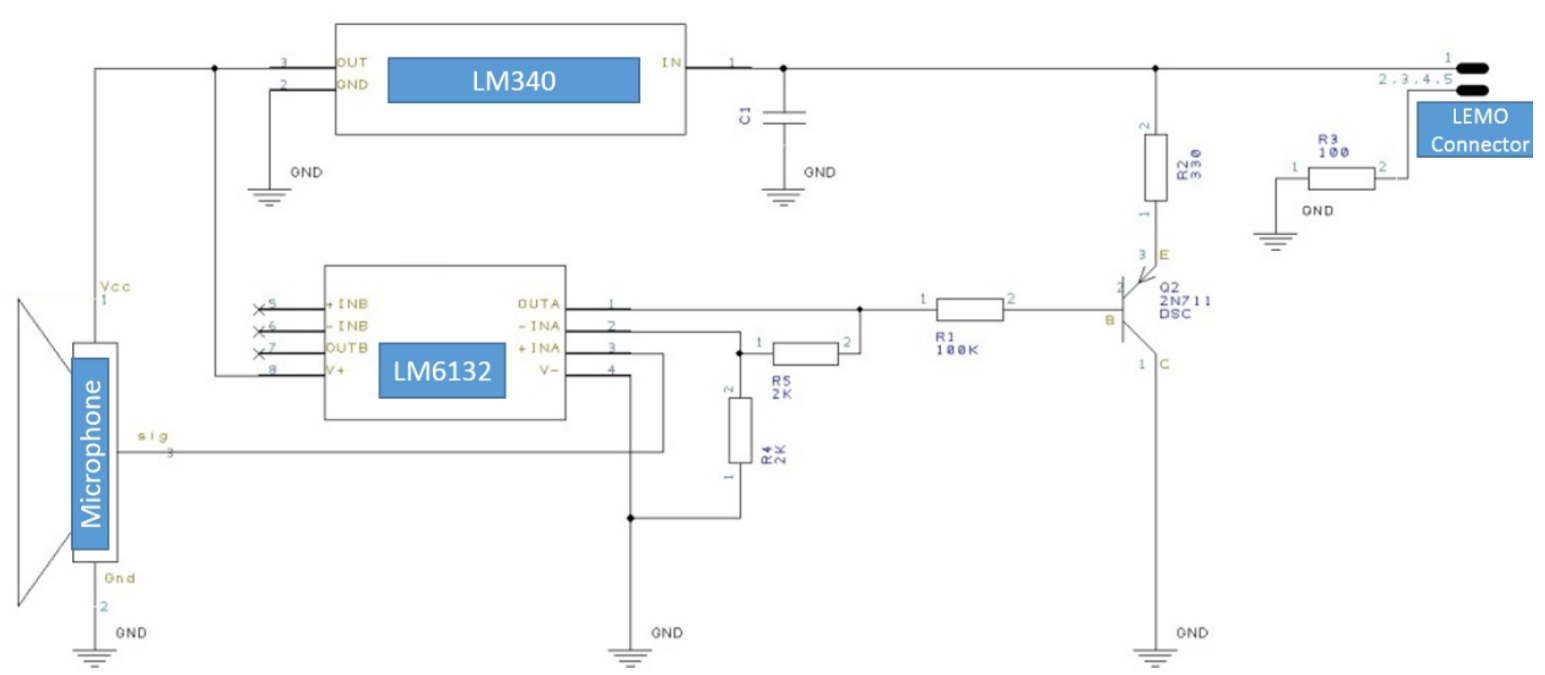

Figure 2. Constant current receiver for array microphones with temperature compensation.

\footnotetext{
* Specific vendor and manufacturer names are explicitly mentioned only to accurately describe the test hardware. The use of vendor and manufacturer names does not imply an endorsement by the U.S. Government nor does it imply that the specified equipment is the best available.
} 


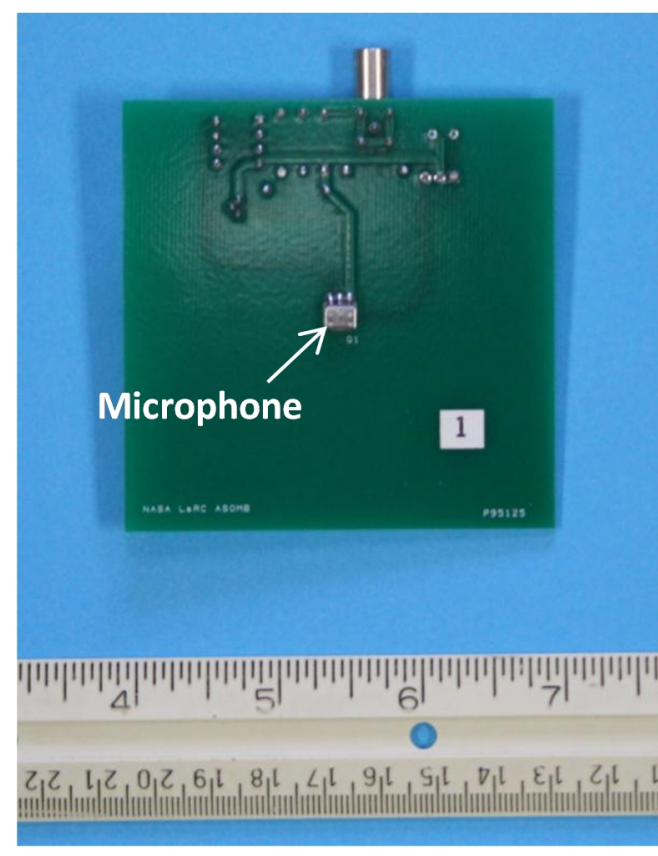

Figure 3. Microphone PCB Front Side.

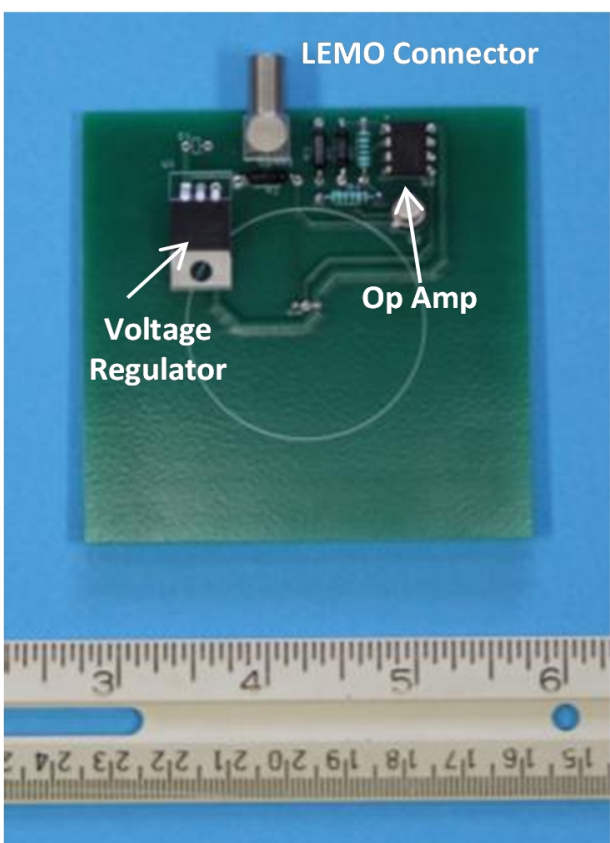

Figure 4. Microphone PCB Back Side.

Microphone Calibration: The sensitivity and frequency response of all the microphones are measured via a benchtop calibration both before and after deployment. The calibrations are conducted using a Bruel and Kjaer 4226 multifunction calibrator as a precision sound source and a National Instruments USB 9215 digitizer to record both the reference signal from the calibrator and the microphone output. Sensitivities are measured using a $250-\mathrm{Hz}, 94-\mathrm{dB}$ sound pressure level tone, corresponding to a pressure excitation of one Pascal at the microphone. Frequency response functions are measured by applying a broadband white noise signal to the external input port of the 4226 calibrator.

Figure 5 shows a representative histogram of the measured sensitivities for the 230 microphones that were fabricated for the array. The distribution of sensitivities is approximately Gaussian in shape with a mean sensitivity of $19.1 \mathrm{mV} / \mathrm{Pa}(-34.4 \mathrm{~dB}$ re $1 \mathrm{~V} / \mathrm{Pa})$ for the ensemble. Microphones with sensitivities at the outer edges of the distribution are identified as spares and are not incorporated into the array unless needed.

Microphone Mounts: Two different types of microphone mounting structures were fabricated for the phased array. The first is a 72-inch diameter central plate populated with the innermost 49 microphones in the array. The plate is manufactured from machined aluminum honeycomb with plastic microphone holders fabricated using an additive manufacturing process. Velcro ${ }^{\circledR}$ strips are used to secure the individual microphone PCB's in the holders. The outer edge of the

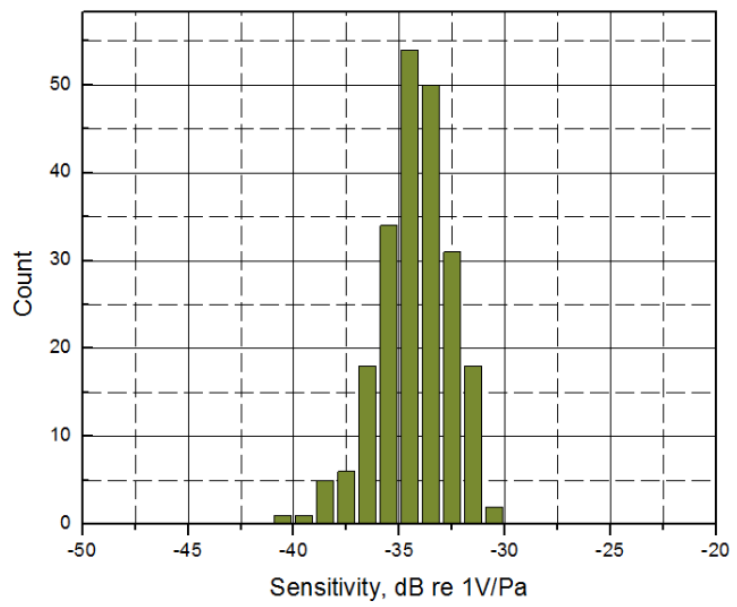

Figure 5. Histogram of measured microphone sensitivities. Mean sensitivity is $19.1 \mathrm{mV} / \mathrm{Pa}$ (-34.4 $\mathrm{dB}$ re $1 \mathrm{~V} / \mathrm{Pa})$. 
central plate is treated with contoured foam to minimize acoustic scattering effects. A close-up of the central plate construction highlighting one of the microphone holders can be seen in Figure 6 . The completed central plate as deployed in the field can be seen in Figure 7. A protective cover is placed over the plate when the array is not in use.

The remaining 136 microphones in the array are mounted on individual 12-inch diameter ground plates manufactured from machined white Plexiglass ${ }^{\circledR}$. Plastic microphone holders identical to those used on the central plate are inserted into the center of each ground plate to secure the microphone PCB's. The edges of the ground plates are contoured to reduce acoustic scattering. Six-inch tall weighted plastic traffic cones are placed over the ground plates when the array is not in operation to protect the sensors from the direct influence of dew and rain. A completed ground plate assembly can be seen in Figure 8.

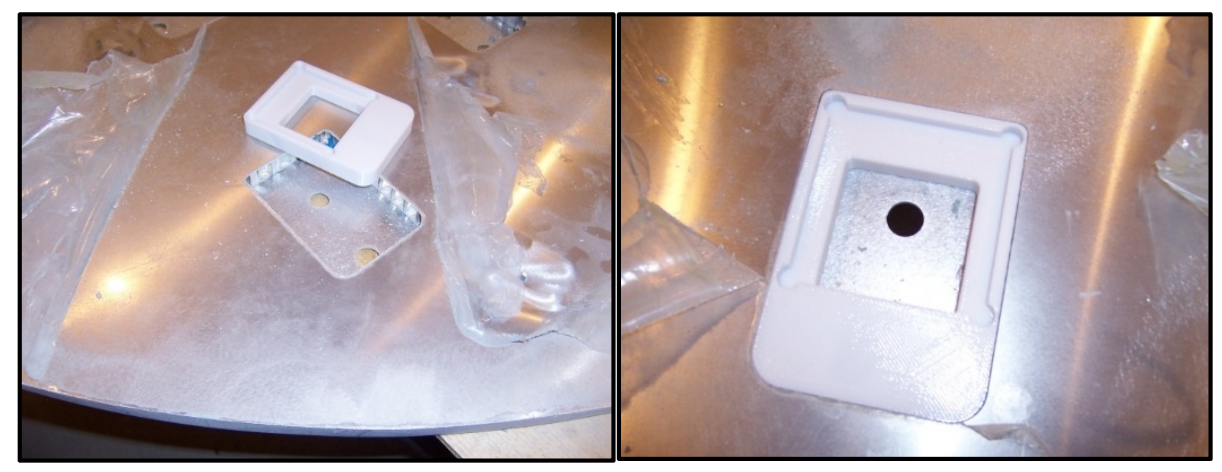

Figure 6. Close-up of central plate construction. Left - Machined aluminum cutout and holder, Right - Assembled unit

Data Acquisition System: A highly distributed data acquisition system was assembled using commercially available hardware. The data acquisition system (built by AVEC, Inc.) has a total capacity of 192 channels and is constructed around General Standards Corporation PMC6616AI64SSA-64-49.152 16-bit synchronous sampling digitizers distributed among three separate PCIe cards. The digitizers are housed in a single high-end computer system with local solid state drive (SSD) disk storage. Signal conditioning of all microphone channels is achieved using an R.C. Electronics DTX-9017 programmable signal conditioner populated with DTX-5290 IEPE plug-in cards (4 channels per card) with fully programmable gain and anti-alias filtering available for each channel. The IEPE cards also supply power to the 4-mA current loops utilized by the microphones. The signal

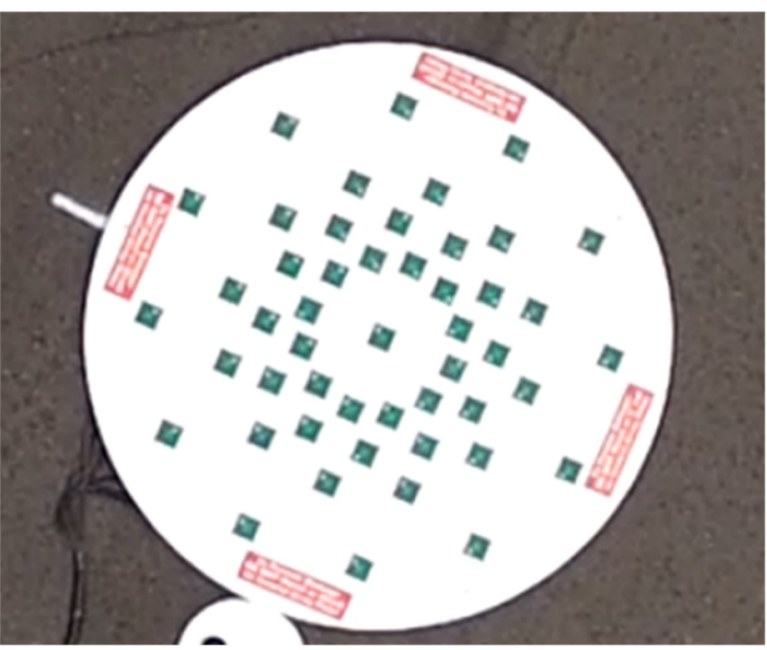

Figure 7. Aerial view of populated central plate. (Video frame from GoPro® ${ }^{\circledR}$ camera) conditioner system is controlled from a master computer which communicates with individual DTX chassis and plug-in cards over a high speed Ethernet connection using software provided by R.C. Electronics. A custom data acquisition module in the AVEC phased array software package ${ }^{*}$ is used for command and control of the General Standards digitizers. Acquired microphone time history data are stored on a high

\footnotetext{
${ }^{*}$ http://www.avec-engineering.com/Products.html [cited April 4, 2016].
} 
capacity network-attached storage (NAS) device as a series of binary data files. The nominal acquisition window length is 30 seconds for a typical run with all channels sampled at $76.8 \mathrm{kHz}$ and lowpass filtered at $20 \mathrm{kHz}$. Finally, an IRIG-B UTC timecode signal is acquired on one acquisition channel on each digitizer card as a sanity check to ensure that synchronization of the system is maintained. The IRIG-B signal also serves as a mechanism to synchronize the array measurements with recorded flight data on test aircraft.

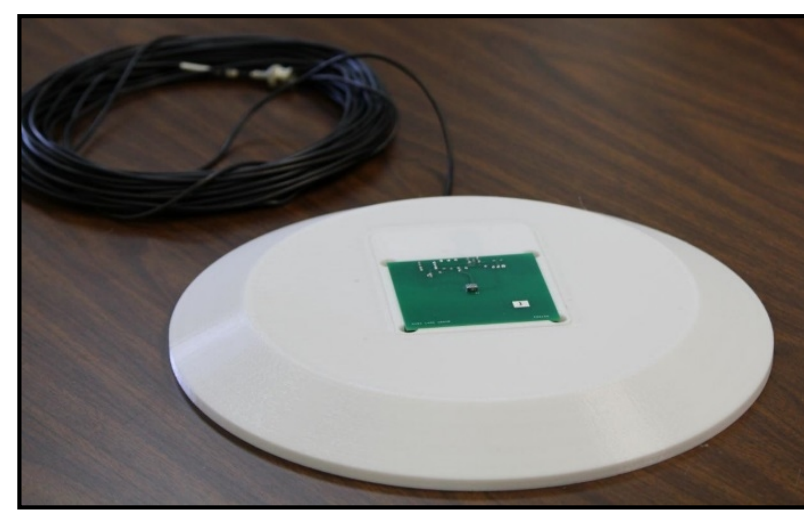

Data Reduction System: The data reduction is performed on a multi-core computer using AVEC's flight test software with custom features jointly developed by AVEC and NASA LaRC. One of the features in the GUI includes beamforming using either AVEC or NASA software routines. The NASA code is based on an original program provided by the Boeing Company, but substantially refactored for the current application (referred to as the BEAMFLY code). Post-processing of the array measurements is conducted using either a conventional delay and sum technique in the time domain or an advanced time domain method described by Dougherty. ${ }^{5}$ A regular Cartesian grid is generated that encompasses and moves with the noise sources on the aircraft. The source location and speed are nominally prescribed by

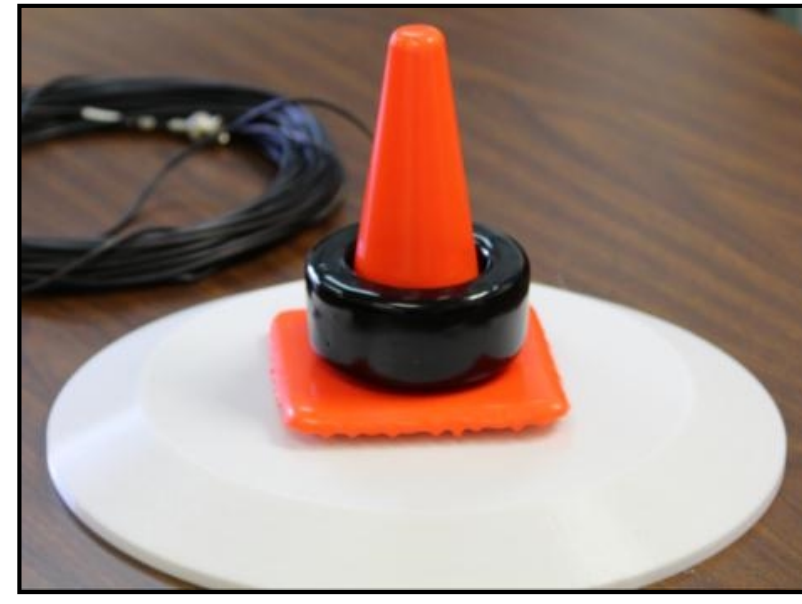

Figure 8. Ground plate assembly. Top - Plate with PCB inserted Bottom - Weighted traffic cone protector differential GPS data describing the position of the aircraft as a function of time. For each potential source location (i.e., grid point), the microphone time histories captured by the array are resampled with time delays and amplitude adjustments corresponding to a monopole moving at locally constant velocity in a homogeneous medium. The "delayed" signals are then summed in such a way that the portions of the signals that conform to a monopole at the grid point under observation sum constructively. Array shading (weighting) is employed with individual microphone weights based on the frequency of interest and the microphone location in the array. The weights effectively reduce the array size as the frequency increases, thereby minimizing decorrelation effects among the outer sensors in the array. Because the beamforming is performed in the time domain, the weights have to be applied during the delay-and-sum process. After applying the shaded delay-and-sum operation, a direct Fourier transform is used to obtain the Fourier coefficients of interest. These coefficients are used in further computations to generate beamform image maps. Atmospheric corrections are applied assuming the air properties and velocities are uniform between the aircraft and the ground, using the absorption model of Bass et al. ${ }^{6}$ Further processing of the data can be performed using deconvolution methods that take into account the actual array response to a moving monopole at each of the source locations. The deconvolution methods available in the AVEC software include the CLEAN-SC and DAMAS algorithms. ${ }^{7-9}$

Support Instrumentation / Hardware: All of the data acquisition and quick-look data analysis hardware for the array system are housed in a command trailer (Figure 9) that can be positioned up to 200 feet away from the microphones (assuming a 200-foot diameter array connected to the trailer using the 400-foot, LEMO- 
terminated cables described previously). The trailer is equipped with a 30-foot weather station that utilizes a sonic anemometer for wind speed and direction, an aspirated temperature / humidity sensor, and a barometric pressure gauge. The weather station performs timestamped sampling of the local conditions on 5-second intervals, with 1-minute averages also computed. The resultant 5-second and 1-minute weather files are stored on the NAS device along with the microphone time histories. The command trailer is also equipped with three surveillance video cameras connected to a digital video recorder (DVR) for monitoring and recording of all aircraft passes over the array. The cameras are attached to pan/tilt gimbal mounts to allow proper orientation of the individual fields of view.

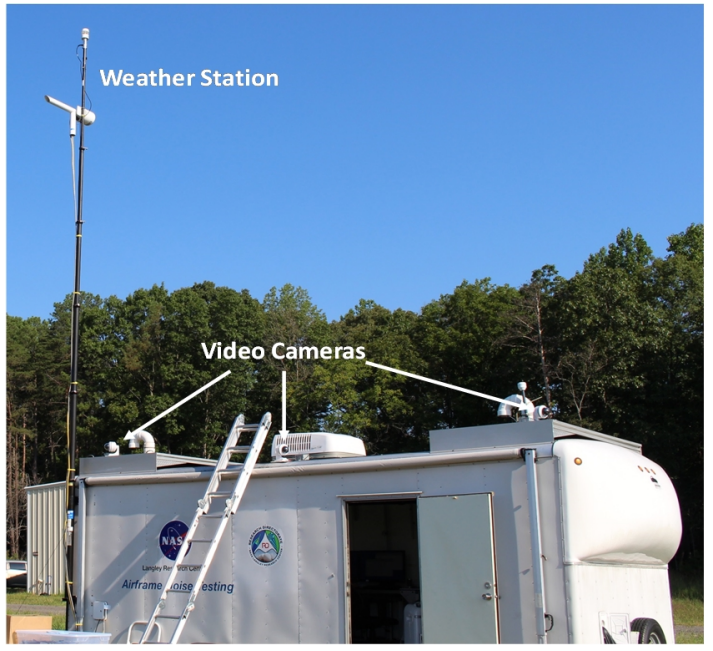

Figure 9. Command trailer for array.

\section{Array Shakedown Testing}

A detailed mock-up of the instrumentation suite (phased array, weather station, and data acquisition and processing) was performed in the LaRC Acoustic Development Laboratory (ADL) prior to the first deployment of the array to: (1) identify any anomalies in the operation of the system that needed correcting, and (2) vet the microphone and overall system performance. Due to space constraints, a simplified and much smaller array pattern than would normally be used was deployed in the test chamber, as shown in Figure 10. Figure 11 depicts the final setup of the array hardware in the facility.

A 1-inch diameter tube connected to a voice-coil speaker driver was suspended approximately 13.5 feet over the center of the array and used as a pseudo monopole point source, exciting the array sensors in turn with tones and white noise. Figure 12 depicts the typical response of the ADL mock-up array when excited

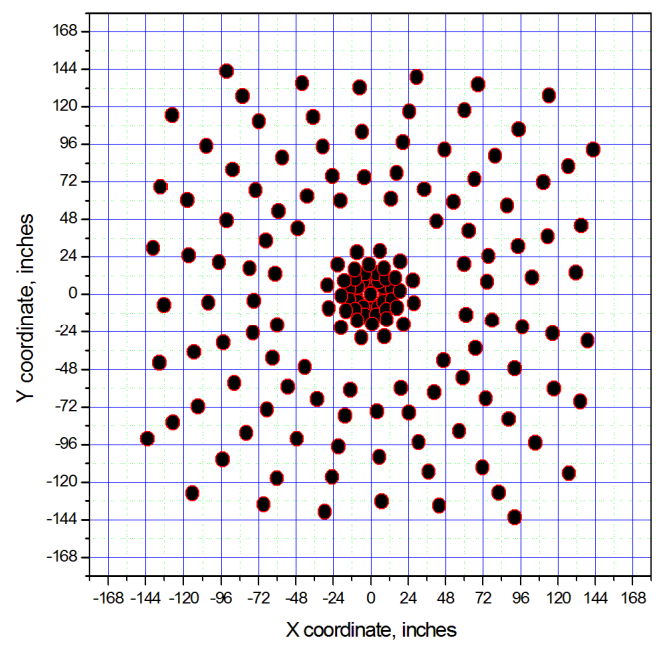

Figure 10. Array pattern deployed in ADL.

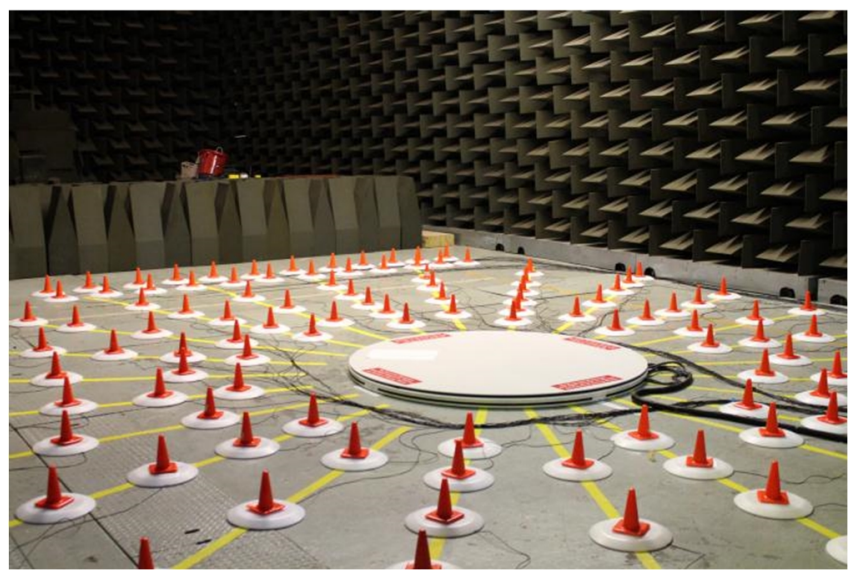

Figure 11. Completed ADL array mock-up. 

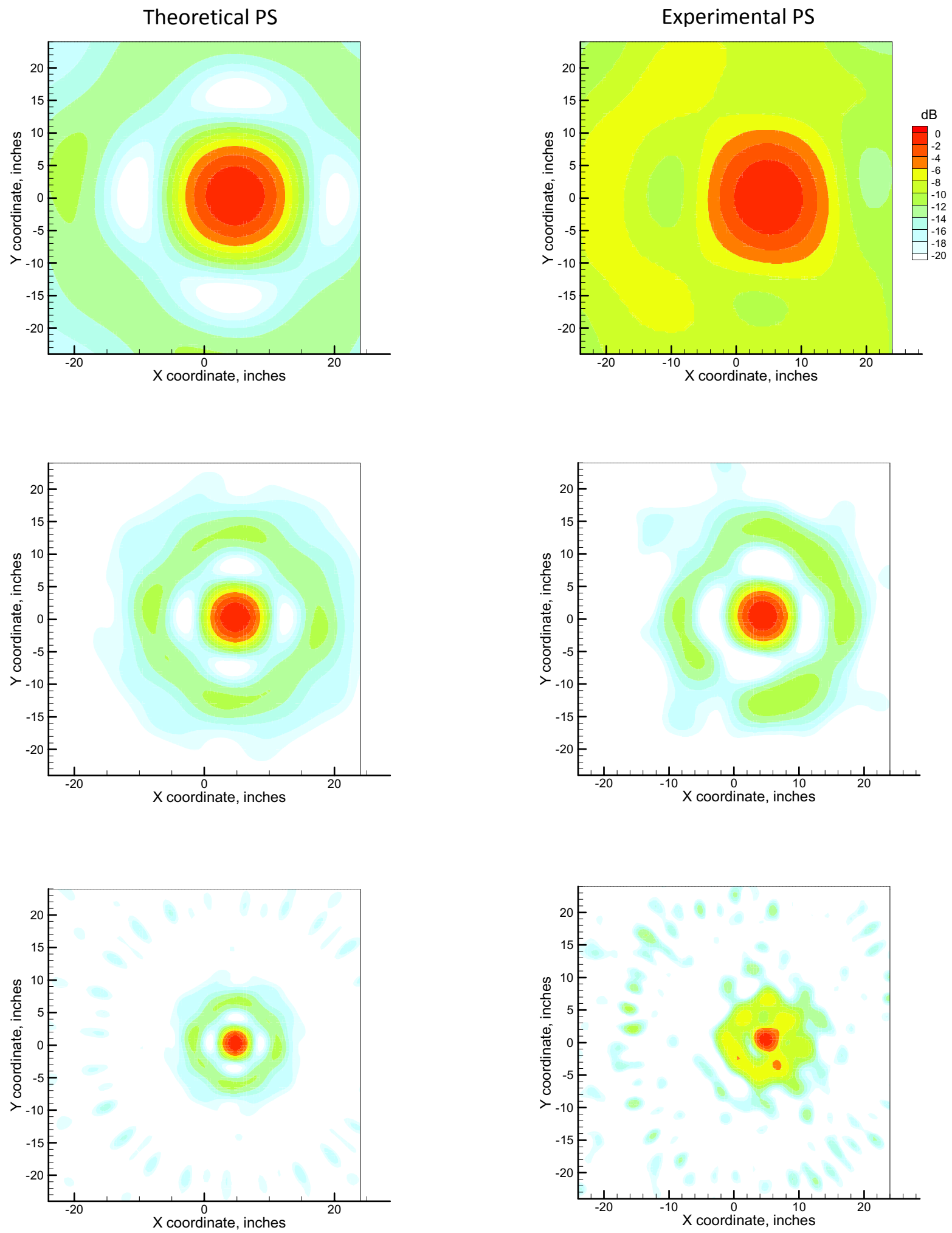

Figure 12. Comparison of narrowband $(\Delta f=75 \mathrm{~Hz})$ beamform maps for theoretical and experimental point sources for array deployment in ADL. Top - 1-kHz Tone, Middle - 2-kHz Tone, Bottom - 4-kHz Tone 
with 1-, 2-, and 4-kHz pure tones. These are compared with theoretical monopole point spread functions computed from the array pattern for like frequencies. In general, good agreement is observed between the theoretical and experimental point source responses of the array. There are higher sidelobe levels observed for the experimental responses versus theory, especially for the 1- and 4-kHz tones. However, it is noted the ADL is only a partially anechoic environment with the sidewalls treated for acoustic reflections and not the ceiling or floor. This can result in an overall degradation of array sidelobe performance. Also, while the central mounting plate was accurately positioned in the test chamber, the locations of the individual ground plates were only accurate to \pm 1 inch, contributing to higher measured sidelobe levels compared with theory.

While in general the array operation was nominal during the ADL mock-up, reliability issues regarding the operation of the data acquisition system were noted and needed to be corrected before the array could be used in an actual deployment. Also, management of the 185 microphone cables was a problem during the mock-up, so improved cable management methodologies were implemented. It was also discovered during the ADL mockup that additional moisture protection was needed for the circuitry on the front and back of the microphone printed circuit boards (refer to Figs. 3 and 4), hence the introduction of the acrylic conformal coating discussed previously.

\section{Initial Deployment of the Array System}

The first deployment of the new phased array system occurred at Finnegan Airfield at Fort A.P. Hill in late August of 2015, where the array was utilized to measure vehicle noise for a number of sUAS (small Unmanned Aerial System) aircraft. The vehicles tested included two Langley J-FLiC (Jet-powered FLying Controls testbed) KingCat jet-powered aircraft (N508NU, N509NU) and a Langley Research Services Directorate (RSD) 40-percent scale Carbon Cub propeller-driven aircraft (N383NA). Also during the deployment, a unique in-situ calibration method for the array microphones using a hovering aerial sound source was attempted for the first time. Only the deployment and calibration of the array using the aerial source will be discussed here. The results of noise measurements on the fixed-wing sUAS aircraft are beyond the scope of the present paper and will be reported on at a later date.

Test Site: Located in Northern Virginia, Finnegan Airfield is a paved sUAS runway approximately 1200 feet long and 75 feet wide with a small slope from the east to west end. It is used for U.S. Army unmanned aircraft system training at Fort A.P. Hill. The runway is operated within restricted airspace and is flanked by tree lines to the north 180 feet away and to the south 200 feet away. A small hangar is located 110 feet to the north of the runway for sUAS aircraft staging and maintenance, and an automated ground weather station (accessible via the internet) is available at the west end of the runway.

Array Pattern and Deployment Details: The array deployed at Finnegan consisted of 185 microphones arranged in an irregular starburst pattern 79 feet wide and 93.7 feet long (Figure 13) and positioned at the east end of the runway with the edge of the array aperture 35 feet from

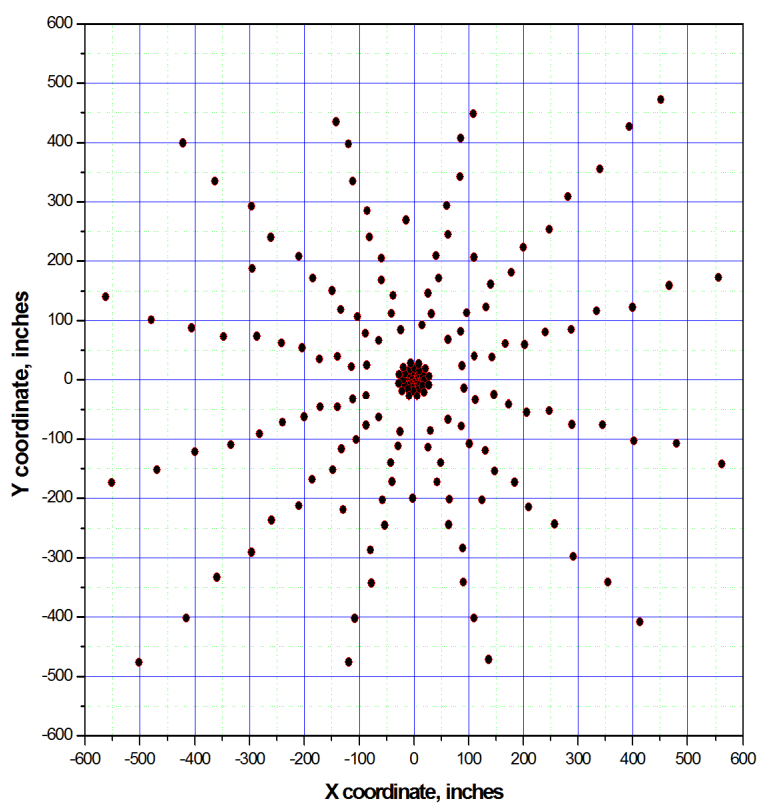

Figure 13. Array pattern deployed at Finnegan. 
the runway threshold. An aerial photo of the deployment is shown in Figure 14. The central 49 microphones of the array were housed on a central mounting plate with the remaining 136 microphones housed on individual ground plates as described in Section II. The array was designed for an operational frequency range of approximately 1-10 kHz. Three additional Bruel and Kjaer freefield microphones were deployed as reference microphones, with one located near the array central mounting plate, one located approximately 70 feet directly up-range of the array along the runway centerline, and one located approximately 70 feet laterally from the edge of the array

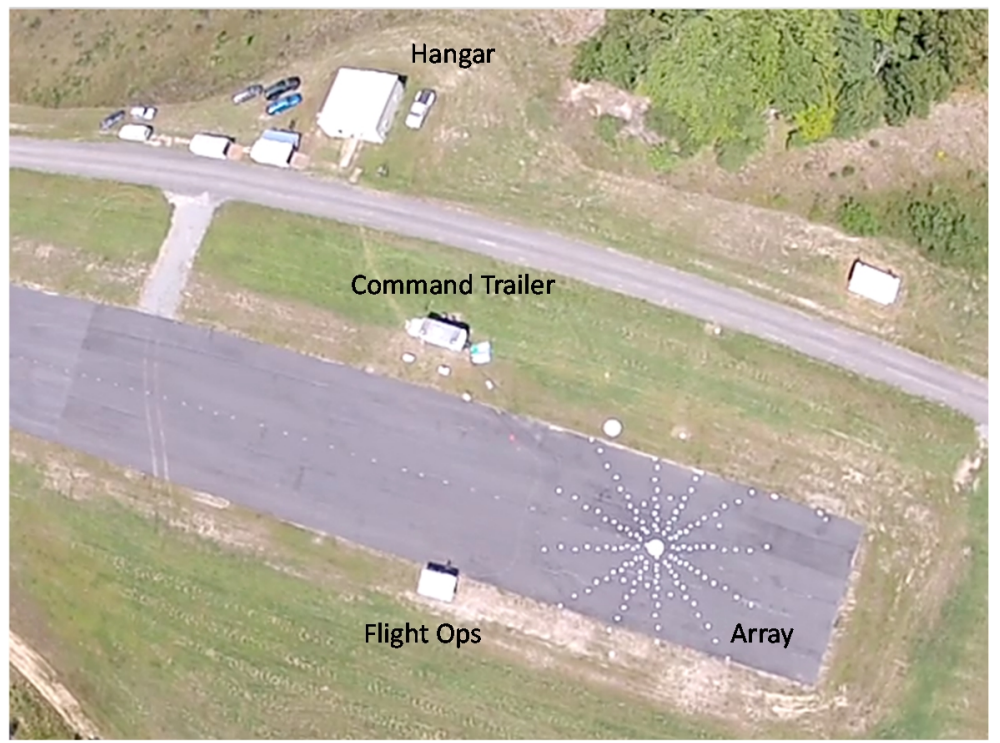

Figure 14. Aerial view of array deployment at Finnegan. on the south side of the runway. The command trailer for the array was positioned in the grass on the north side of the runway approximately 75 feet from the edge of the array aperture.

Calibration of Array: A unique in-situ calibration method for the array sensors was attempted for the first time during the deployment using a hovering aerial sound source. The vehicle chosen for the calibrations was a Prioria Robotics Hex Flyer provided by the LaRC Autonomy Incubator (AI) with a suspended battery-powered Anchor ${ }^{\circledR}$ MiniVox Lite public address speaker and MP3 player as the payload. These are shown in flight in Figure 15. The speaker provided both tonal and white noise excitation of the array at a number of vehicle altitudes spanning 50 to 400 feet.

In principle, comparison of recorded microphone output levels during the Hex Flyer flights allows the relative sensitivity drift of the sensors to be tracked on a daily basis, as long as the effects of speaker directivity, sound propagation (including wind) and vehicle station keeping are taken into account. In order to understand the effect of speaker directivity on the local sound pressure levels recorded by the array microphones, a detailed characterization of the speaker response was performed in the LaRC Structural Acoustics Loads and Transmission (SALT) facility. Figure 16 shows details of the SALT testing. The facility consists of adjacent reverberation and anechoic test chambers where structural test panels can be acoustically excited and measured. However, only the anechoic test chamber was utilized for the speaker characterization. This chamber has dimensions of $15 \times 25 \times 31.5$ feet and is covered with 36-inch acoustic wedges. The aerial speaker was placed 86.5 inches above the test chamber floor on a rotation stage (see top panel of Fig. 16). The speaker was tested in two orientations on the stage - upright for azimuthal

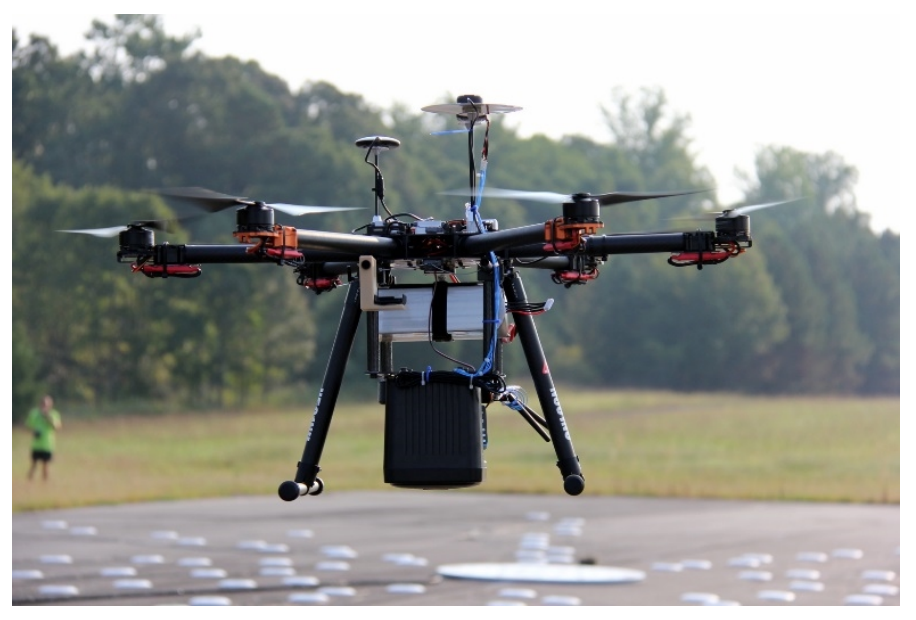

Figure 15. Close up of Langley Hex Flyer in flight with aerial speaker mounted directly underneath. 

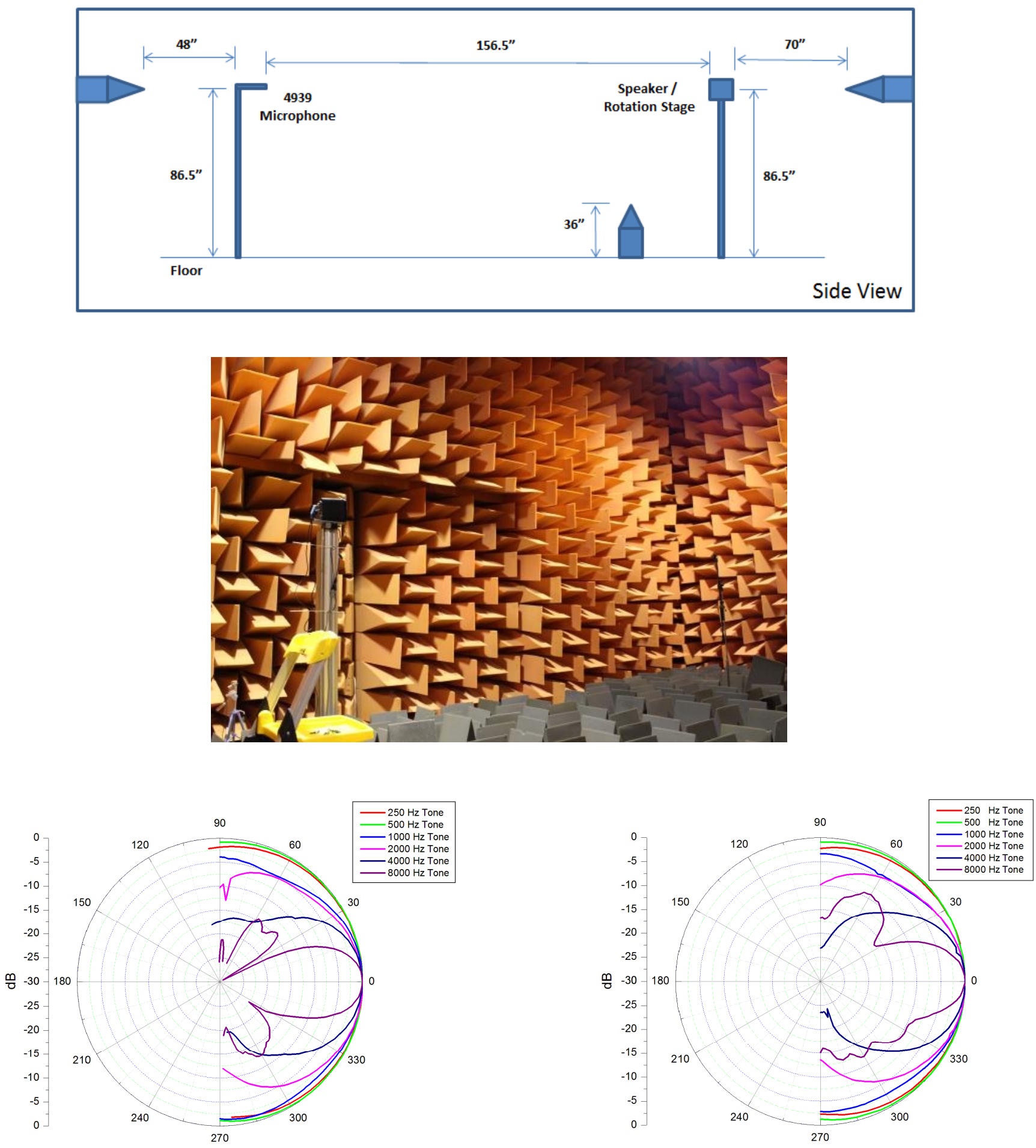

Figure 16. Aerial sound source directivity characterization in SALT. Top - Test Setup

Middle - SALT Test Chamber Showing Mounted Speaker (Left of Center in Photo) Bottom - Directivity Functions for Polar (Left) and Azimuthal (Right) Orientations 
(horizontal plane) measurements, and on its side for polar (vertical plane) measurements. A Bruel and Kjaer 4939 freefield microphone was positioned 156.5 inches in front of the speaker at the same height above the floor. The microphone was sampled at a $20 \mathrm{kHz}$ sampling rate with data acquired for 5 seconds for each angular position of the speaker. Measurements of speaker directivity were performed over an angular range of \pm 90 degrees in both the azimuthal and polar orientations to obtain hemispherical response functions. Speaker angular increments of 2 or 5 degrees were chosen depending on the narrowband frequency of interest.

The bottom left panel of Fig. 16 depicts hemispherical speaker directivities as a function of polar angle for pure tones ranging from $250 \mathrm{~Hz}$ to $8 \mathrm{kHz}$. The amplitudes have been normalized to $0 \mathrm{~dB}$ at 0 degrees. As expected, a narrowing of the directivity and the introduction of speaker sidelobes are observed in the data as the frequency increases. In general the speaker is well behaved below $2 \mathrm{kHz}$, with a very uniform and flat directivity observed below $1 \mathrm{kHz}$. Strong sidelobe structures are introduced into the directivity at $8 \mathrm{kHz}$. Very similar directivity characteristics are observed as a function of azimuthal angle in the bottom right panel of Fig. 16, although the sidelobe structure at $8 \mathrm{kHz}$ is broader in the azimuthal direction versus the polar direction.

In order to predict the effects of the speaker directivity on measured SPL levels given the array aperture deployed at Finnegan, the maximum $\mathrm{dB}$ loss at those angles corresponding to the edges of the aperture in the polar and azimuthal directions were extracted from the directivity functions shown in Fig. 16. These are tabulated in Table 1, and assume a diameter for the array of 79 feet and vertical altitudes for the Hex Flyer of 100, 200, and 400 feet.

Table 1. Predicted dB loss at Edge of Array Due to Sound Source Directivity

\begin{tabular}{|c|c|c|c|c|c|c|}
\hline & \multicolumn{2}{|c|}{ Altitude $=\mathbf{1 0 0}$ feet } & \multicolumn{2}{c|}{ Altitude = 200 feet } & \multicolumn{2}{c|}{ Altitude = 400 feet } \\
\hline $\begin{array}{c}\text { Frequency } \\
(\mathbf{H z})\end{array}$ & $\begin{array}{c}\text { Polar dB } \\
\text { Loss }\end{array}$ & $\begin{array}{c}\text { Azimuthal } \\
\text { dB Loss }\end{array}$ & $\begin{array}{c}\text { Polar dB } \\
\text { Loss }\end{array}$ & $\begin{array}{c}\text { Azimuthal } \\
\text { dB Loss }\end{array}$ & $\begin{array}{c}\text { Polar dB } \\
\text { Loss }\end{array}$ & $\begin{array}{c}\text { Azimuthal } \\
\text { dB Loss }\end{array}$ \\
\hline 250 & 0.1 & 0.05 & 0.04 & 0.01 & 0.01 & 0.004 \\
\hline 500 & 0.1 & 0.07 & 0.05 & 0.04 & 0.04 & 0.03 \\
\hline 1000 & 0.8 & 1.1 & 0.2 & 0.4 & 0.1 & 0.2 \\
\hline 2000 & 1.3 & 1.0 & 0.6 & 0.3 & 0.2 & 0.2 \\
\hline 4000 & 2.2 & 2.1 & 0.6 & 0.5 & 0.3 & 0.1 \\
\hline 8000 & 8.6 & 6.8 & 1.8 & 1.8 & 0.6 & 0.6 \\
\hline
\end{tabular}

An examination of the $\mathrm{dB}$ loss values in Table 1 shows that the best performance of the aerial speaker is at altitudes above 200 feet for frequencies below $4 \mathrm{kHz}$, with a maximum predicted loss of only $0.6 \mathrm{~dB}$. It is noted that the majority of Hex Flyer flights performed at Finnegan utilized $4 \mathrm{kHz}$ tones and white noise excitation to calibrate the array. Although not tested in the SALT, it is reasonable to assume that driving the speaker with white noise will also exhibit acceptable directivity performance at higher altitudes. Therefore, a conclusion can be made that speaker directivity will not be a major contributor to errors in the calibrations if the vehicle is maintained at altitudes exceeding 200 feet.

Similar to the speaker directivity characterization, it is instructive to examine the Hex Flyer vehicle stability and station keeping during calibration runs. It is important for the vehicle to maintain a stationary position over the array at a prescribed altitude to provide a stable sound source. The Hex Flyer incorporated a differential GPS tracking system manufactured by Swift Navigation that provided real-time monitoring of vehicle location, velocity, and angles during the flights. Figure 17 shows examples of the vehicle positional stability as a function of altitude for flights made on August 27, 2015 at Finnegan. The data for each of these flights spans approximately 30 seconds. It was expected that the vehicle station keeping would become more difficult as the altitude increases, presumably due to an increase in winds at higher altitudes. The drift shown in Fig. 17 in the $\mathrm{x}$ and y coordinates for the vehicle are not extreme, with maximum deviations of one foot or less in the x position and two feet or less in the y position. However, the deviations in the vehicle $\mathrm{z}$ coordinate are much more a function of the absolute altitude, with deviations 
of two feet shown at the 100 foot altitude, but six and four feet shown at the 200 and 400 foot altitudes, respectively. Unfortunately a weather balloon was not utilized at Finnegan, so only winds at the 30-foot level were available. Nevertheless, given the proximity of the tree lines lateral to the runway, it is reasonable to assume that wind speeds and perhaps shear increases at altitudes above the tree line. Finally,
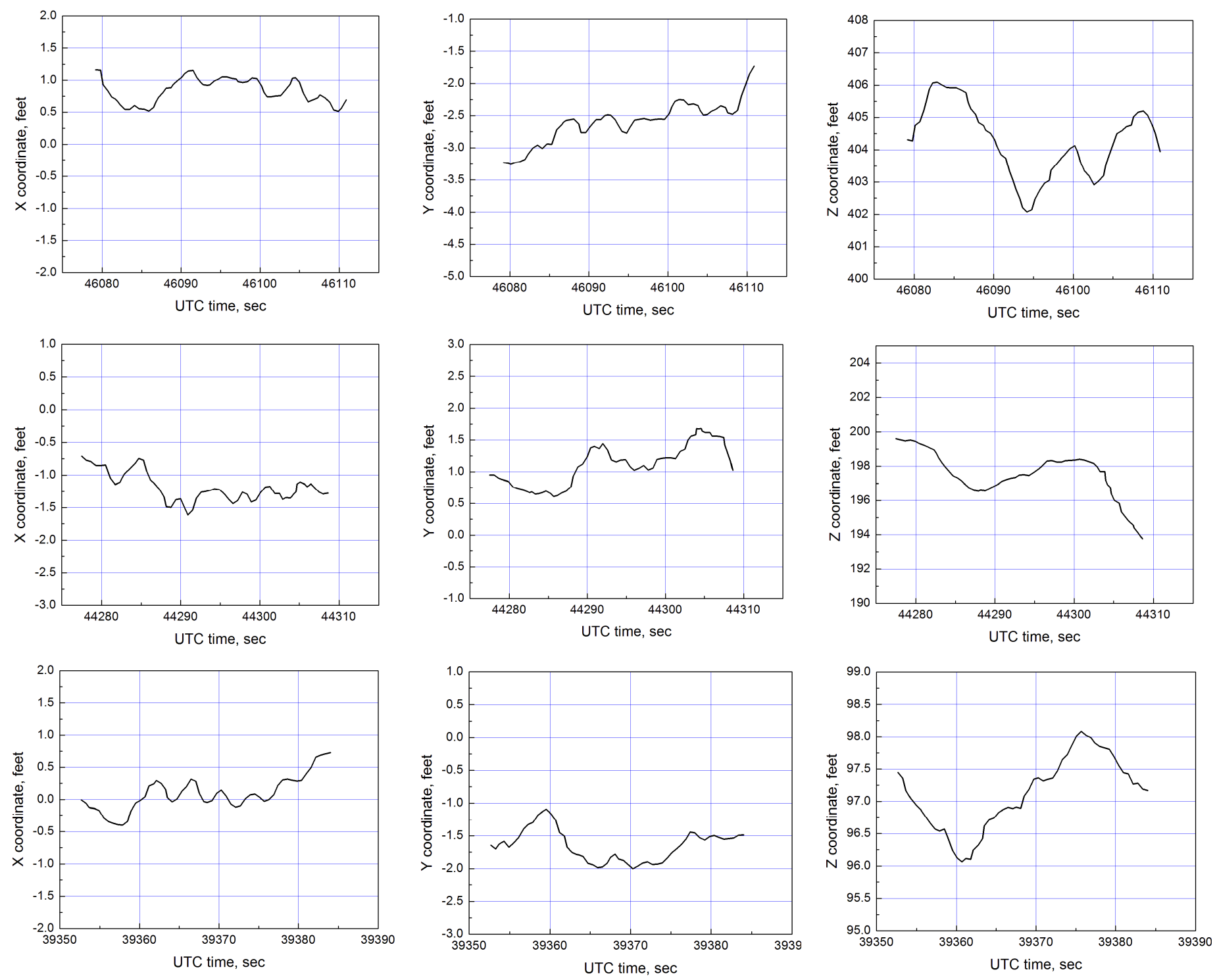

Figure 17. Representative Hex Flyer $x, y, z$ tracking data provided by on-board differential GPS unit. Top - 400-foot Altitude, Middle - 200-foot Altitude, Bottom - 100-foot Altitude

an examination of the pitch and roll angles for the vehicle show maximum angular deviations of less than 5-7 degrees in most cases for the Hex Flyer flights conducted on August 27. Overall, an examination of the GPS data indicates that the overall stability of the vehicle was reasonable. However, as will be seen, an increase in station keeping requirements for the vehicle at altitude can result in increased rotor noise as the vehicle works to maintain position.

Using information derived from the speaker directivities shown in Fig. 16 and vehicle tracking data like that shown in Fig. 17, a series of tabulated microphone levels and noise footprints across the array aperture were created and used to track the daily performance of the sensors during the deployment. This is illustrated by example in Figure 18 using data obtained from aerial speaker measurements conducted on

13 of 20

American Institute of Aeronautics and Astronautics 

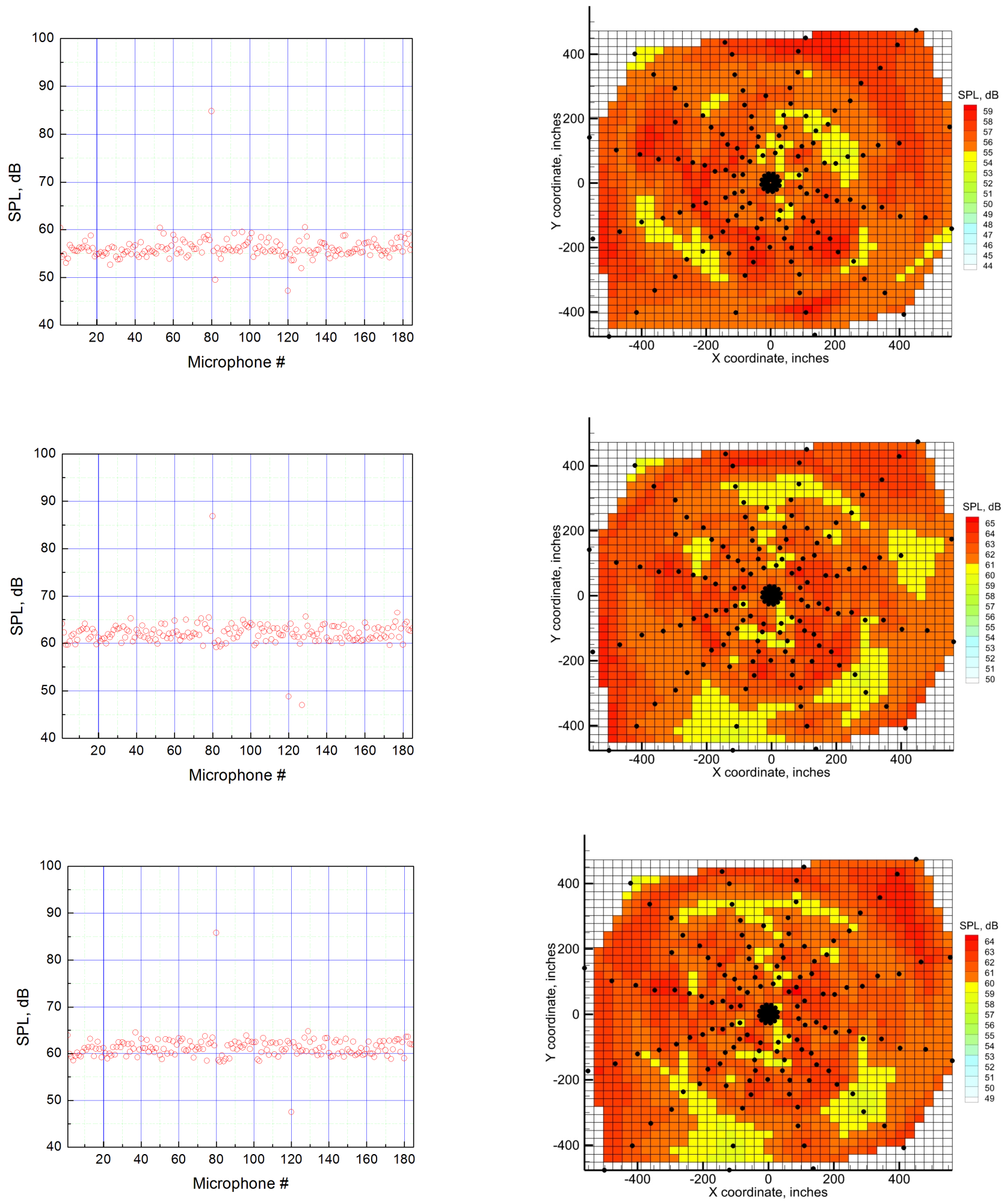

Figure 18. Measured microphone levels and ground footprints for Hex Flyer calibration flight conducted on August 27, 2015. Vehicle altitude - 400 feet. Top - 4-kHz tone calibration, Middle - white noise calibration, Bottom - vehicle noise only (no speaker) 

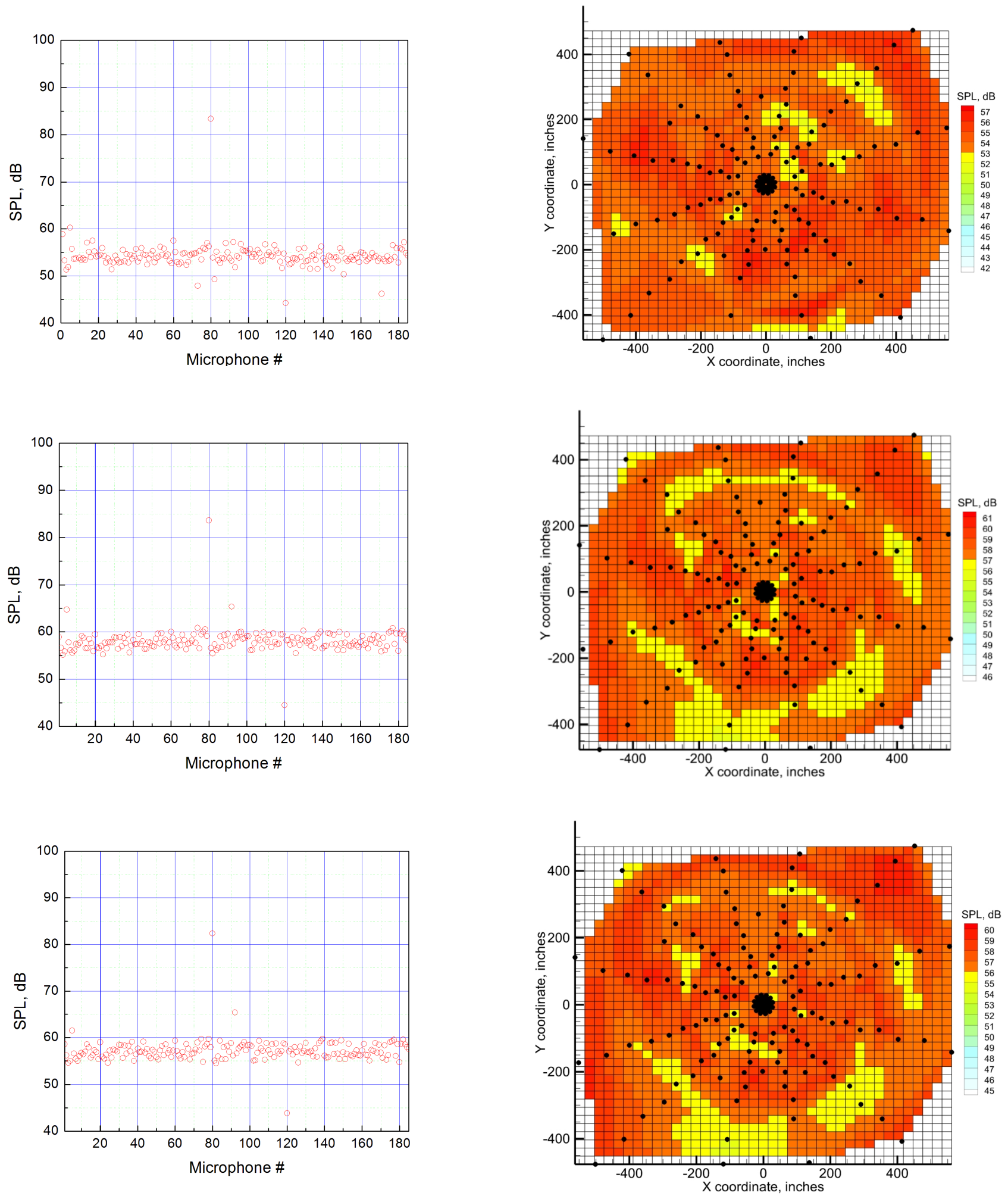

Figure 19. Measured microphone levels and ground footprints for Hex Flyer calibration flight conducted on September 2, 2015. Vehicle altitude - 400 feet. Top - 4-kHz tone calibration, Middle - white noise calibration, Bottom - vehicle noise only (no speaker) 
August 27 and in Figure 19 for like measurements conducted on September 2. In both figures, the top two panels represent narrowband measurements $(\Delta f=37.5 \mathrm{~Hz})$ for a $4-\mathrm{kHz}$ pure tone signal applied to the aerial speaker. The middle two panels represent measurements for a white noise signal, while the bottom two panels are with the speaker disabled (vehicle noise only). For the white noise and vehicle noise cases, the narrowband auto-spectra for the array microphones have been summed (on a pressure-squared basis) over a frequency range of $5-10 \mathrm{kHz}$. The ground footprint contours are obtained via a linear interpolation of either the narrowband or summed levels recorded by the microphones, projected onto a uniformly-spaced Cartesian grid. All of the data have been corrected for an assumed uniform atmospheric propagation based on the temperature measured at 30 feet. The data have also been corrected for mean vehicle position and altitude as reported by the GPS tracking. Note that microphones 80 and 120 in the array were bad, and thus have been excluded from the noise footprint contours.

Several conclusions can be drawn from an examination of the data shown in the figures:

1. There is some scatter shown in the microphone levels and ground footprints for the 4-kHz pure tone calibrations, with very similar amounts of scatter shown between Figs. 18 and 19. The reason for this scatter is not totally understood. The aerial speaker directivity functions shown in Fig. 16 do show a much smaller main directivity lobe at $4 \mathrm{kHz}$ than those at lower frequencies. As a result, it would be expected that changes in yaw and pitch angles as a result of vehicle station keeping would degrade the measured acoustic levels at the edges of the array aperture. However, level changes on the order of 5-6 dB are observed both near the center of the array and midway out along the array arms in roughly diagonal directions. It is speculated that scattering from the Hex Flyer landing rails (which have a diameter of roughly 1 inch and extend below the level of the aerial speaker) might be responsible for these level changes. Acoustic scattering from the ground plates themselves might also contribute to the observed level changes.

2. The ground footprints for the white noise calibrations also exhibit scatter, but are remarkably similar between Figs. 18 and 19, even though the measurements were taken six days apart. This is actually an encouraging finding, and implies that the calibrations using the aerial speaker will be viable. Note that while the white noise contributions to the microphone autospectra are small with the sound source at 400 feet, they are still measurable and provide what appears to be a stable calibration.

3. The ground footprints observed when only the Hex Flyer vehicle noise was measured (no aerial sound source) are very similar to those shown for the white noise calibrations. This reinforces the conclusion that at 400 feet the ground noise is dominated by the vehicle with only a small contribution from the aerial source. Nevertheless, very consistent ground footprints are evident for the calibration runs on the two days.

Figure 20 shows a series of $\Delta d B$ differences in measured microphone levels for four different representative microphones across the array aperture, extracted from white noise calibration runs performed over a six-day period spanning August 27 through September 2. Microphones 1 and 2 are adjacent ones at the middle of the central mounting plate while microphones 58 and 140 are on ground plates on roughly opposite sides of the array. In order to correct for absolute differences in the levels due to changes in speaker volume settings (which had to be adjusted manually before calibration flights), the global mean levels observed across the array were subtracted from each day's data before the $\Delta d B$ values were computed. The plots shown in Fig. 20 have been normalized to the levels measured by the microphones on day 1.

As can be seen in Fig. 20, over the six-day period, the maximum drifts in recorded levels were $-2 \mathrm{~dB},-2.5 \mathrm{~dB},-1.3 \mathrm{~dB}$, and $-1.7 \mathrm{~dB}$ for microphone $1,2,58$, and 140 , respectively. Of note is the downward slope shown in the data of Fig. 20, especially for the microphones mounted on the central plate. The reason for this slope is not known at this time, and microphone laboratory calibrations conducted both before and after the deployment did not reveal significant differences in measured sensor sensitivities. Further, no diaphragm aging problems were observed for the sensors. Regardless, the observed levels for

16 of 20

American Institute of Aeronautics and Astronautics 

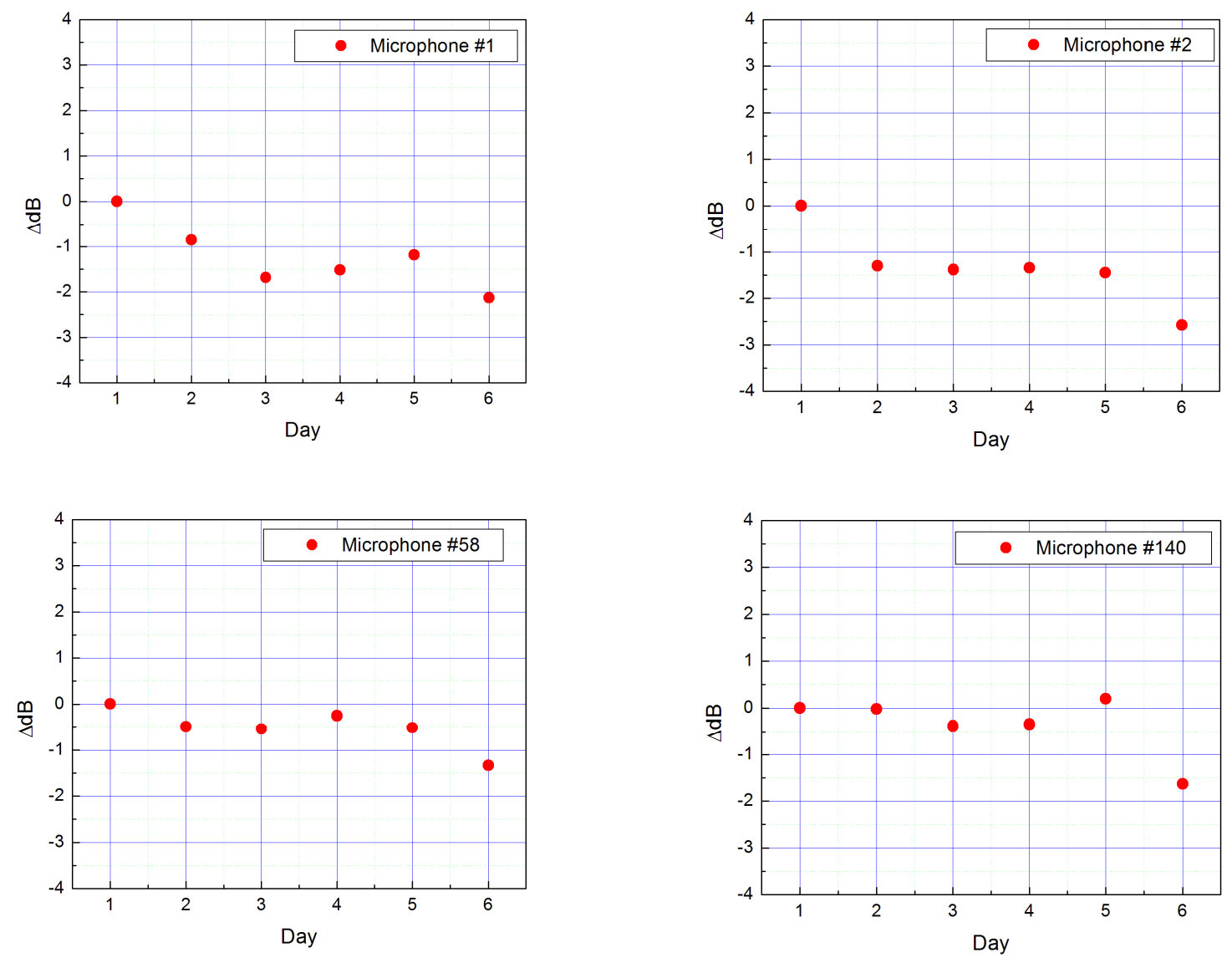

Figure 20. Early-morning measured microphone levels for 6-day period spanning August 27 through September 2.

Microphones 1, 2 on central plate. Microphones 58, 140 on ground plates. Hex Flyer altitude $=\mathbf{4 0 0} \mathrm{ft}$, White noise calibration.

days 2 through 5 are remarkably uniform. This uniformity is reflected in the ground footprint contours of Figs. 18 and 19, where the contour patterns are virtually identical over the six-day period shown. It is noted that only 8 microphones out of 185 exhibited problems requiring replacement with spare units during the deployment, despite the sensors being subjected to extremes of temperatures up to $100^{\circ} \mathrm{F}$ and relative humidity up to $100 \%$.

Finally, Figure 21 shows a series of beamform noise contour plots for the Hex Flyer at a number of altitudes with the aerial sound source generating either a 4-kHz pure tone or white noise. The noise contours shown in Fig. 21 have been corrected for atmospheric propagation, but only assuming a uniform atmosphere with the conditions observed at the 30 -foot high weather station. As a consequence, the peak noise levels are only approximate. Furthermore, the amplitudes have not been normalized to account for the vehicle altitude. Nevertheless, the time-domain beamformer described in Section II does an adequate job of capturing and visualizing the speaker noise, particularly for the pure tone runs. For the white noise runs, more of the rotor noise can be observed interfering with the speaker noise, particularly for higher vehicle altitudes where the white noise levels are comparable to the rotor noise levels. This finding agrees with the similarly observed microphone levels shown in Figs. 18 and 19 for the white noise and vehicle noise-only runs, with the increase in the overall noise caused by the addition of the white noise being small. Note also that ideally the noise should attenuate based on acoustic spherical spreading as the vehicle altitude increases. While there is some decrease in the measured levels for the higher altitudes depicted in Fig. 21, 

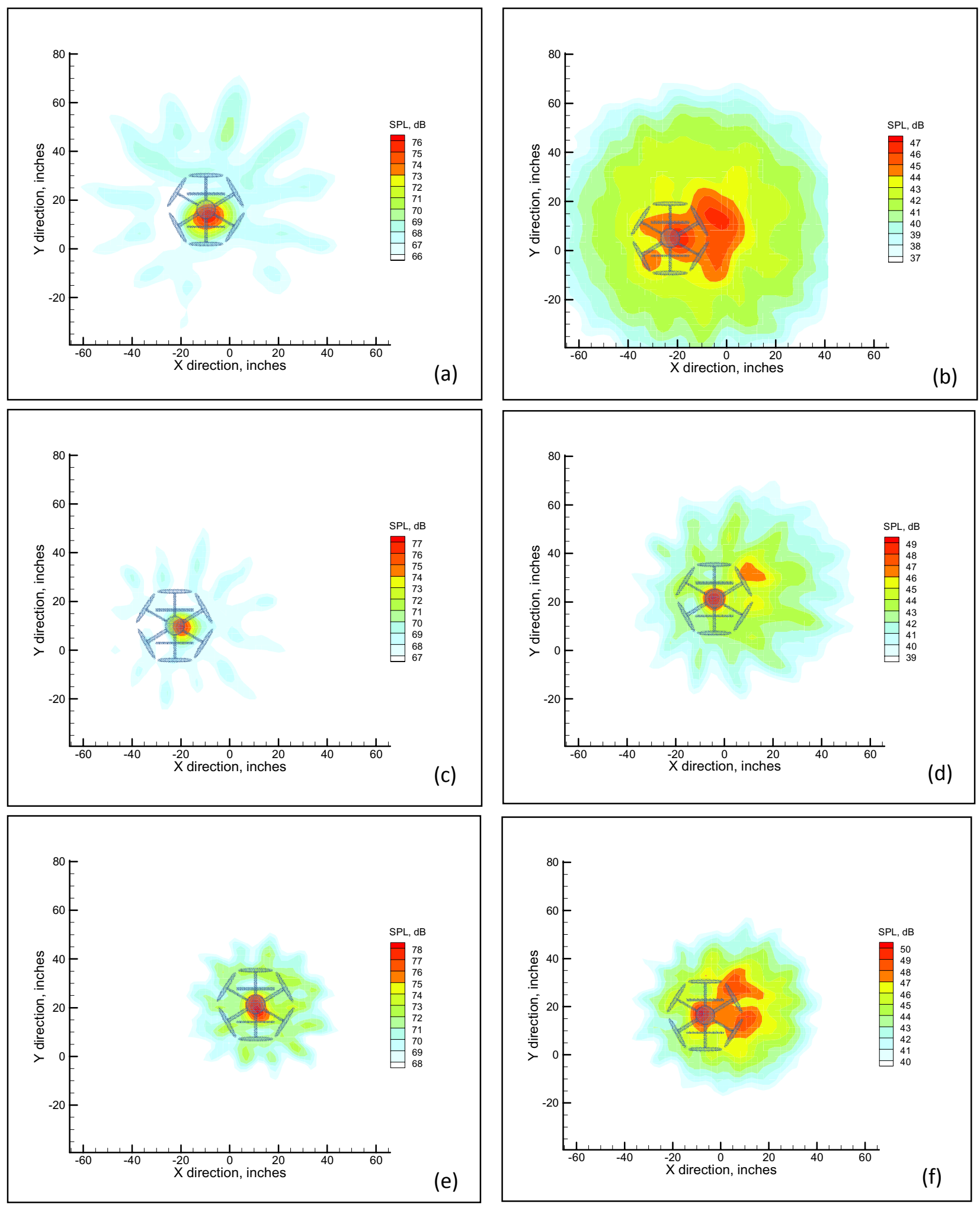

Figure 21. 3975-Hz Narrowband $(\Delta f=75 \mathrm{~Hz})$ noise contours for Hex Flyer calibration flight.

(a) - 4-kHz tone, 100-foot altitude, (b) - white noise, 100-foot altitude

(c) - 4-kHz tone, 75-foot altitude, (d) - white noise, 75-foot altitude

(e) - 4-kHz tone, 50-foot altitude, (f) - white noise, 50-foot altitude 
it is speculated that the approximated propagation and wind effects may be causing the observed levels to be higher than expected. Another contributor could again be related to vehicle station keeping, with variable or higher rotor noise at higher altitudes.

\section{Future Improvements}

Based on the lessons learned from the operation of the phased array at Finnegan Airfield, a number of improvements are currently being implemented to the system prior to a planned deployment at the NASA Armstrong Flight Research Center in 2016. These improvements include the replacement of the Hex Flyer vehicle with a new DJI S1000 octocopter. This vehicle has a higher payload capacity with similar flight durations as the Hex Flyer. A higher payload capacity is required since the previous fixed aerial speaker mount will be replaced with a heavier active gimbal mount (similar to that shown in Figure 22) to improve pointing stability of the speaker as the vehicle performs station keeping. The mount is designed for aerial cameras but will be modified for use with the speaker.

In order to improve the quality of the in-situ calibrations performed on the array, in additional to the aerial sound source, a series of ground-based calibration speakers will be placed throughout the array pattern. The speakers, consisting of dome tweeters, will be flush-mounted on ground plates similar to those used for the microphones. The speakers will be operated sequentially one at a time with separate sets of data acquired by the array microphones. The daily recorded microphone levels for the ground sources as well as the aerial source will be tracked using similar procedures to those described in Section IV.

\section{Summary}

A new aeroacoustic measurement capability has been developed for use in measurement of airframe and propulsive noise for a range of aircraft types and scales. The instrumentation consists of a large channel-count, field-deployable microphone phased array plus support hardware. The array incorporates up to 185 hardened, weather-resistant sensors suitable for extended outdoor deployment. A custom 4-mA current loop receiver circuit with temperature compensation was developed to power the sensors over extended cable lengths with minimal degradation of the signal to noise ratio and frequency response. A compact data system combining sensor power, signal conditioning, and digitization was assembled for use with the array, as were a commercial weather station and a video monitoring / recording system. The array system was thoroughly tested in the Langley Acoustic Development Laboratory prior to an initial deployment at Fort A.P. Hill to measure the noise generated by a range of sUAS aircraft. During this deployment, a new calibration method for the array using a hovering aerial sound source was attempted for the first time, with the results indicating that daily drifts in microphone sensitivities can be tracked, assuming vehicle station keeping is stable. The array operation during the deployment was nominal with remarkable stability observed for the microphones, and the hardware is now available for use on-demand for future flight tests. 


\section{Acknowledgments}

The authors gratefully acknowledge the work of William D. Castle of the Langley Aeronautics Systems Engineering Branch and Glenn D. Ormsby of Science and Technology Corporation in fabricating the mounting components for the phased array and preparing the command trailer for deployment at Fort A.P. Hill. This work was funded by the NASA Environmentally Responsible Aviation (ERA) Project under Integrated Technology Demonstration activity (ITD) 50A.

\section{References}

${ }^{1}$ Collier, F. S., "Environmentally Responsible Aviation (ERA) Project," oral presentation, NASA Fundamental Aeronautics Program, Third Annual Meeting, Atlanta, GA, Sept., 2009.

${ }^{2}$ Khorrami, M. R., Humphreys, W. M., Jr., Lockard, D. P., and Ravetta, P. A., “Aeroacoustic Evaluation of Flap and Landing Gear Noise Reduction Concepts,", AIAA Paper 2014-2478, $20^{\text {th }}$ AIAA/CEAS Aeroacoustics Conference, Atlanta, GA, 2014.

${ }^{3}$ Humphreys, W. M., Jr., Brooks, T. F., Hunter, W. W., Jr., and Meadows, K. R., "Design and Use of Microphone Directional Arrays for Aeroacoustic Measurements," AIAA Paper 1998-0471, 36st Aerospace Sciences Meeting and Exhibit, Reno, NV, 1998.

${ }^{4}$ Khorrami, M. R., Lockard, D. P., Humphreys, W. M., Jr., Choudhari, M. M., and Van de Ven, T., "Preliminary Analysis of Acoustic Measurements from the NASA-Gulfstream Airframe Noise Flight Test," AIAA Paper 2008-2814, 14 ${ }^{\text {th }}$ AIAA/CEAS Aeroacoustics Conference, Vancouver, Canada, 2008.

${ }^{5}$ Dougherty, R., “Advanced Time-Domain Beamforming Techniques,” AIAA-2004-2955, $10^{\text {th }}$ AIAA/CEAS Aeroacoustics Conference, Manchester, UK, May 10-12, 2004.

${ }^{6}$ Bass, H. E., Sutherland, L. C., Zuckerwar, A. J., Blackstock, and D. T., Hester, D. M., "Atmospheric absorption of sound: Further developments," Journal of the Acoustical Society of America, 97(1), pp. 680683, January, 1995.

${ }^{7}$ Guerin, S., and Siller, H., "A Hybrid Time-Frequency Approach to the Noise Localization Analysis of Aircraft Flyovers," AIAA-2008-2955，14 ${ }^{\text {th }}$ AIAA/CEAS Aeroacoustics Conference, Vancouver, Canada, May 5-7, 2008.

${ }^{8}$ Brooks, T. F., and Humphreys, W. M., Jr., “A Deconvolution Approach for the Mapping of Acoustic Sources (DAMAS) Determined from Phased Microphone Arrays", Journal of Sound and Vibration, Volume 294, 2006, pp. 856-879.

${ }^{9}$ Sijtsma, P., "CLEAN Based on Spatial Source Coherence," AIAA-2007-3436, $13^{\text {th }}$ AIAA/CEAS Aeroacoustics Conference, Rome, Italy, 2014. 